\title{
Reactive Oxygen Species Modulator I As An Adverse Prognostic Marker In Stage III Non-Small Cell Lung Cancer Treated With Radiotherapy: A Retrospective Pilot Study
}

This article was published in the following Dove Press journal:

OncoTargets and Therapy

\author{
Moonkyoo Kong (D) \\ Ji-Youn Sung ${ }^{2}$ \\ Seung Hyeun Lee $\mathbb{D}^{3}$ \\ 'Division of Lung \& Head and Neck \\ Oncology, Department of Radiation \\ Oncology, Kyung Hee University Medical \\ Center, Kyung Hee University School of \\ Medicine, Seoul, Republic of Korea; \\ ${ }^{2}$ Department of Pathology, Kyung Hee \\ University Medical Center, Kyung Hee \\ University School of Medicine, Seoul, \\ Republic of Korea; ${ }^{3}$ Division of \\ Pulmonary and Critical Care Medicine, \\ Department of Internal Medicine, Kyung \\ Hee University Medical Center, Kyung \\ Hee University School of Medicine, \\ Seoul, Republic of Korea
}

Purpose: Reactive oxygen species modulator 1 (ROMO1) is a novel protein regulating intracellular reactive oxygen species production. Although increased ROMO1 expression has been associated with poor clinical outcomes in several human malignancies, the clinical implication of this protein in a radiotherapy setting has never been explored. The aim of this study was to investigate whether ROMO1 expression is associated with survival in lung cancer patients who received radiotherapy.

Methods: ROMO1 protein expression was evaluated immunohistochemically using histologic score (H-score) in 49 tumor tissues from stage III non-small cell lung cancer (NSCLC) patients treated with definitive radiotherapy. We performed survival analyses according to various clinicopathological parameters including ROMO1 expression.

Results: ROMO1 expression was not associated with any clinicopathological parameter of age, sex, smoking status, stage, or histological subtype. Multivariate analyses showed that high ROMO1 expression was independently associated with worse progression-free survival (hazard ratio [HR] = $1.87,95 \%$ confidence interval [CI]: $1.02-4.23)$ and with worse overall survival $(\mathrm{HR}=2.79,95 \%$ CI:1.13-6.87). In addition, high ROMO1 expression was independently associated with shorter time to loco-regional recurrence $(\mathrm{HR}=2.71,95 \% \mathrm{CI}: 1.04-6.28)$ but was not associated with time to distant metastasis.

Conclusion: ROMO1 overexpression was associated with early loco-regional recurrence and poor survival outcomes in stage III NSCLC treated with definitive radiotherapy. Our exploratory results provide a basis for further large-scale studies to validate whether ROMO1 could be a prognostic marker in this setting.

Keywords: lung cancer, radiotherapy, reactive oxygen species, ROMO1, survival, biomarker

\section{Introduction}

Lung cancer is the leading cause of cancer-related death globally. ${ }^{1-3}$ Approximately 220,000 new cases of lung cancer and 158,000 cases of lung cancer-related deaths occurred in the United States in 2015. ${ }^{4}$ Although, advances in targeted therapy and immunotherapy have demonstrated remarkable survival benefits for metastatic nonsmall cell lung cancer (NSCLC), there is relatively modest progress in treatment of locally advanced disease, i.e., stage III NSCLC. Based on the established superiority of concurrent chemoradiotherapy (CRT) over sequential treatment in
Correspondence: Seung Hyeun Lee Division of Pulmonary and Critical Care Medicine, Department of Interna Medicine, Kyung Hee University School of Medicine, Kyungheedae-ro 23,

Dongdaemun-gu, Seoul 02447, Republic of Korea

Tel +822958 851 I

Fax +8229681848

Emailv3mann@naver.com 
inoperable stage III NSCLC, ${ }^{5}$ many trials have been conducted to evaluate the clinical benefit of new chemotherapeutic agents or consolidation treatment. However, these trials have failed to demonstrate prolonged survival compared to conventional regimens, and the 5-year survival rate of stage III NSCLC remains $27-32 \%{ }^{6-8}$ Therefore, discovery and clinical application of biomarkers are crucial in improving the survival of patients by predicting treatment outcome and optimizing or individualizing the current treatment strategies for high-risk patients.

ROMO1 is a novel protein that has been discovered in human head and neck cancer tissue. It is located in the mitochondrial inner membrane, where it controls mitochondrial reactive oxygen species (ROS) production. ${ }^{9}$ Earlier studies have demonstrated that ROMO1 is up-regulated in a variety of human cancer cells, and that ROMO1-induced ROS production is essential for proliferation of cancer cells, as well as normal cells. ${ }^{9}$ Recent clinical studies have shown the prognostic value of ROMO1 expression in several human malignancies. ROMO1 overexpression was associated with tumor invasiveness and poor survival in hepatocellular carcinoma patients who received surgical resection. ${ }^{10}$ Similar results were reported for colorectal cancer patients. ${ }^{11}$ Recently, we have reported that ROMO1 expression was associated with shorter survival in NSCLC patients who were treated with platinum-based chemotherapy and surgical resection. ${ }^{12,13}$ These previous studies suggest that ROMO1 confers tumor aggressiveness or invasiveness and thus can be used as a potential biomarker. Considering that ROS-induced DNA damage is a critical step in radiotherapy (RT)-induced cancer cell death, ROMO1, a key regulator of ROS production, could be closely associated with resistance to RT, as shown in chemotherapy and surgical resection.

Based on this hypothesis, we conducted the present study to investigate whether ROMO1 expression is associated with treatment outcome in inoperable stage III NSCLC patients who received definitive RT and whether ROMO1 has predictive or prognostic value in this clinical setting.

\section{Materials And Methods}

\section{Study Subjects And Data Collection}

We retrospectively recruited patients who received RT for treatment of lung cancer at Kyung Hee University Hospital, a tertiary referral hospital in South Korea, from
January 2010 to July 2017. Criteria for inclusion in this study were histologically confirmed stage III non-small cell lung cancer, receipt of definitive RT with or without chemotherapy, Eastern Cooperative Oncology Group (ECOG) performance status $\leq 2$, available cancer tissue specimens, no previous history of thoracic RT, no previous or concurrent illness that would compromise completion of treatment, and available follow-up data. Patients who received postoperative or palliative RT were excluded.

All the patients underwent staging work-up including chest computed tomography (CT), brain magnetic resonance imaging, and ${ }^{18} \mathrm{~F}$-fluorodeoxyglucose positron emission tomography (PET). TNM staging was determined according to the 8th edition of the International Association for the Study of Lung Cancer TNM staging system. We reviewed electronic medical records to obtain demographic information, past medical or social history, and clinical data of all participants. This study protocol was approved by the Clinical Research Ethics Committee of Kyung Hee University Hospital, and written informed consent was obtained from all live patients but was waived for deceased patients. All research was carried out in compliance with the Declaration of Helsinki.

\section{Radiotherapy Schedule}

All patients received CT-planned RT. The gross extent of the primary tumor and grossly involved lymph nodes visualized on chest CT and PET were target lesions of RT. Elective nodal irradiation was not performed. The prescription dose was determined by a radiation oncologist (M Kong) based on size of the target lesion, patient's general condition, and probability of RT-induced toxicity. A daily dose of 1.8-2.5 Gy was delivered at five fractions per week, resulting in a total dose of 57.5-70.4 Gy. For standard comparison of different RT dose schedules, biologically equivalent doses were calculated using a linear quadratic model with an $\alpha / \beta$ ratio of 10 . Implementation and regimen of chemotherapy were individualized based on patient performance status and compliance. Follow-up visits were scheduled 1 month after completion of RT and every 2-3 months thereafter. At follow-up visits, basic laboratory studies, chest radiography, and chest CT scan were conducted. PET was also performed as needed. 


\section{Quantification Of ROMOI Protein Expression}

Formalin-fixed paraffin-embedded tumor tissue specimens were used for evaluation of ROMO1 protein expression by immunohistochemical staining. Staining was performed on 4- $\mu \mathrm{m}$-thick tissue sections using the Bond Polymer Refine Detection System (Leica Biosystems, Newcastle, UK) according to the manufacturer's instructions. Representative paraffin blocks were selected after initial evaluation of hematoxylin-eosin stained slides. Sections were deparaffinized with Bond Dewax Solution (Leica Biosystems), and antigen retrieval was performed using Bond Epitope Retrieval Solution 1 (Leica Biosystems) for $20 \mathrm{mins}$ at $100^{\circ} \mathrm{C}$. Endogenous peroxidase was quenched by incubation with hydrogen peroxide for 15 mins. Sections were incubated in a Bond-Max Immunoautostainer (Leica Biosystems) for 15 mins with 1:200 dilution of ROMO1 mouse monoclonal antibody (OTI2C12, OriGene, Rockville, MD, USA) labeled with a biotin-free polymeric horseradish peroxidase-linker antibody conjugate system. Bound peroxidase was visualized using a solution of diaminobenzidine as the chromogen, and nuclei were counterstained with Mayer's hematoxylin. Colonic adenocarcinoma and normal breast tissue were used as external positive controls, and endothelial cells around the tumor were used as an internal positive control. All sections were examined by a pathologist (JY Sung) who was blinded to the clinical data. Sections were examined under a light microscope at $\times 200$, and cytoplasmic staining was considered positive expression. Staining intensity was defined as follows: 0 , no staining; 1 , weak; 2 , moderate; or 3, strong. Quantification of positivity $(0-100 \%)$ was based on an estimate of the percentage of tumor cells with the specific staining intensity. The final histologic scores (H scores) were obtained by the following equation: $\mathrm{H}$ score $=$ (proportion of tumor cells with no staining $\mathrm{x} 0$ ) + (proportion of tumor cells with weak intensity $\mathrm{x} 1)+$ (proportion of tumor cells with moderate intensity $\times 2$ ) + (proportion of tumor cells with strong intensity $\mathrm{x} 3$ ). The final $\mathrm{H}$ scores range from 0 to 300 .

\section{Statistical Analyses}

For discriminating between low and high ROMO1 expression, the cutoff $\mathrm{H}$-score was defined as the point with the lowest $p$-value on the log rank test for all possible $\mathrm{H}$-scores. To assess the association between ROMO1 expression and clinical outcome, we analyzed time to loco-regional recurrence (TLR), time to distant metastasis (TDM), progression-free survival (PFS), and overall survival (OS).
Loco-regional recurrence was defined as an increase in size of target lesions or appearance of new lesions in the ipsilateral thorax, ipsilateral and/or contralateral hilum, mediastinum, and supraclavicular lymph node regions, while distant metastasis was defined as evidence of tumor in any other area. PFS and OS were defined as the times from the first day of treatment to disease progression/death and to death from any cause, respectively. Baseline characteristics between groups were compared using Fisher's exact test. Associations between clinical parameters and survival were first evaluated by univariate analysis using log rank test; subsequently, multivariate Cox's proportional hazard regression was conducted with adjustment for parameters with $p$ values less than 0.3 in univariate analysis. Survival curves were generated using the Kaplan-Meier method. $P$ values less than 0.05 were considered significant. All analyses were performed using SPSS version 21.0 (IBM Corporation, Armonk, NY, USA).

\section{Results \\ Patient Characteristics}

Among the total of 213 patients who received RT for treatment of lung cancer at our institution during the screening period, 156 did not meet the inclusion criteria of this study; 60 patients showed histologically confirmed small cell lung cancer, 45 patients received postoperative or palliative RT, 21 patients were not stage III, 18 patients did not have available cancer tissue specimens, and 12 patients were ECOG performance status $\geq 3$. Among the remaining 57 patients, 8 whose tissues were unsuitable for immunohistochemical staining were also excluded. Therefore, 49 patients were finally included in this study.

Clinical characteristics of the study population are summarized in Table 1. All were Korean, and the median age was 72 years (range, 49 to 80 years). Twenty-nine patients $(59 \%)$ were over 70 years of age, and 8 patients $(16 \%)$ were female. Thirty-three patients (67\%) had squamous cell carcinoma, and 29 patients (59\%) had stage IIIA disease. Thirty-six patients (73\%) had an Eastern Cooperative Oncology Group (ECOG) performance status of 0 or 1, and 26 patients $(53 \%)$ were current smokers. Fifteen patients $(31 \%)$ were treated with intensity-modulated RT (IMRT), while $34(69 \%)$ were treated with 3-dimensional conformal RT (3D-CRT). The median biologically equivalent dose (BED) was $75.1 \mathrm{~Gy}_{10}$ (range, 70.1-82.5 Gy 10 ). Ten patients (20\%) experienced temporary RT interruption due to treatment-related toxicities, and the median duration 
Table I Clinical Characteristics Of Patients According To Romol Expression (N=49)

\begin{tabular}{|c|c|c|c|c|}
\hline & \multirow{2}{*}{ No. Of Patients (\%) } & \multicolumn{2}{|l|}{ Romol Expression } & \multirow[t]{2}{*}{$p$-value } \\
\hline & & Low $(H-S c o r e ~ \leq 200)(n=35)$ & High $(H-S c o r e ~>200)(n=14)$ & \\
\hline Age (years) & & & & 0.453 \\
\hline$<70$ & $18(37)$ & 14 & 4 & \\
\hline$\geq 70$ & $31(63)$ & 21 & 10 & \\
\hline Sex & & & & 0.672 \\
\hline Male & $41(84)$ & 30 & 11 & \\
\hline Female & $8(16)$ & 5 & 3 & \\
\hline Histology & & & & 0.501 \\
\hline SQCC & $33(67)$ & 25 & 8 & \\
\hline ADC & $16(33)$ & 10 & 6 & \\
\hline Stage & & & & 0.408 \\
\hline IIIA & $29(59)$ & 22 & 7 & \\
\hline IIIB & $20(4 I)$ & 13 & 7 & \\
\hline ECOG performance & & & & 0.297 \\
\hline $0-1$ & $36(73)$ & 24 & 12 & \\
\hline 2 & $13(27)$ & 11 & 2 & \\
\hline Smoking status & & & & 0.319 \\
\hline Current & $26(53)$ & 17 & 9 & \\
\hline Former or never & $23(47)$ & 18 & 5 & \\
\hline Radiotherapy technique & & & & 0.502 \\
\hline IMRT & $15(3 \mid)$ & 12 & 3 & \\
\hline 3D-CRT & $34(69)$ & 23 & 11 & \\
\hline Total dose (BED, Gy,0) & & & & 0.248 \\
\hline Median (range) & $49(100)$ & $76.2(70.1-84.0)$ & $74.3(70.1-80.5)$ & \\
\hline Daily dose (Gy) & & & & 0.364 \\
\hline Median (range) & $49(100)$ & $2.1(1.8-2.5)$ & $2.0(1.8-2.2)$ & \\
\hline Radiotherapy interruption & & & & 0.597 \\
\hline Yes & $10(20)$ & 7 & 3 & \\
\hline No & $39(80)$ & 28 & 11 & \\
\hline Chemotherapy & & & & 0.276 \\
\hline Yes & $29(59)$ & 20 & 9 & \\
\hline No & $20(4 I)$ & 15 & 5 & \\
\hline Types of Chemotherapy* & & & & 0.872 \\
\hline Sequential & II (38) & 8 & 3 & \\
\hline Concurrent & $18(62)$ & 12 & 6 & \\
\hline
\end{tabular}

Notes: *Types of chemotherapy were analyzed in 29 patients who received chemotherapy.

Abbreviations: ECOG, Eastern Cooperative Oncology Group; ADC, adenocarcinoma; SQCC, squamous cell carcinoma; IMRT, intensity-modulated radiotherapy; 3D-CRT, 3-dimensional conformal radiotherapy; BED, biologically equivalent dose.

of RT interruption was 5 days (range, 4-17 days). Chemotherapy was performed in 29 patients (59\%); concurrent and sequential in $18(62 \%)$ and 11 patients (38\%), respectively. Etoposide/platinum, vinorelbine/platinum, and paclitaxel/platinum was used as chemotherapy in 14 (48\%), 10 (34\%), and 5 patients (18\%), respectively. 


\section{ROMOI Expression}

Representative sections with different $\mathrm{H}$-scores are shown in Figure 1. ROMO1 protein was mainly localized in the cytoplasm of cancer cells, which is consistent with previous studies. ${ }^{10,13}$ The ROMO1 H-scores were normally distributed with a median of 155 (range, 10-295).

\section{Associations Between ROMOI Expression And Clinicopathological Parameters}

The optimal cutoff $\mathrm{H}$-score for discriminating between low and high ROMO1 expression was determined to be 200 . With this cutoff, 35 patients $(71 \%)$ were allocated to the low ROMO1 group and 14 patients (29\%) to the high ROMO1 group.

To evaluate whether ROMO1 expression is associated with clinicopathological parameters, we compared the proportions of patients in different patient groups according to ROMO1 expression. However, there was no significant difference in patient or tumor characteristics between the low and high ROMO1 expression groups, suggesting that clinicopathological parameters of age, sex, smoking status, performance status, stage, and histological subtype are not associated with ROMO1 expression (Table 1).

\section{Associations Between ROMOI Expression And PFS And OS}

The median follow-up duration of all patients was 21.0 months (range, 4.0-80.0 months). Results for PFS and OS according to clinicopathological parameter are summarized in Table 2. The median PFS for the overall population was 10.4 months (range, 2.2-80.0 months). Univariate analysis showed that advanced age, current smoking, and high ROMO1 expression showed a non-significant decreasing trend for PFS. In the multivariate analysis, current smoking (HR=2.03, 95\% CI: $1.01-4.46)$ was significantly associated with shorter PFS. In addition, high ROMO1 expression was independently associated with shorter PFS (HR=1.87, 95\% CI:1.02-4.23). The KaplanMeier survival curves also showed that patients with high ROMO1 expression were likely to have shorter PFS (Figure 2A).

During the follow-up period, $18(18 / 35,51.4 \%)$ and 9 patients $(9 / 14,64.3 \%)$ died in the low and high ROMO1 expression groups, respectively. The median OS for all study subjects was 24.3 months (range, 3.5-80.0 months). Univariate analysis revealed that poor performance status (17.0 versus 32.5 months, $p=0.046)$ and high ROMO1 expression (13.5 versus 29.0 months, $p=0.038)$ were significantly associated with shorter OS. Multivariate analysis showed that poor performance status ( $\mathrm{HR}=2.37,95 \%$ CI:1.08-6.55) was significantly associated with shorter OS. In addition, high ROMO1 expression was independently associated with shorter OS (HR=2.79, 95\% CI:1.13-6.87). The Kaplan-Meier survival curves also showed that patients with high ROMO1 expression were likely to have a shorter OS (Figure 2B).

\section{Associations Between ROMOI Expression And TLR And TDM}

During the follow-up period, loco-regional recurrence occurred in $14(14 / 35,40.0 \%)$ and 8 patients $(8 / 14$, $57.1 \%$ ) in the low and high ROMO1 expression groups, respectively. In addition, $13(13 / 35,37.1 \%)$ and 6 patients $(6 / 14,42.9 \%)$ experienced distant metastases in each of these groups, respectively. Among the 22 patients who experienced loco-regional recurrences, 12 (12/22, 54.5\%)

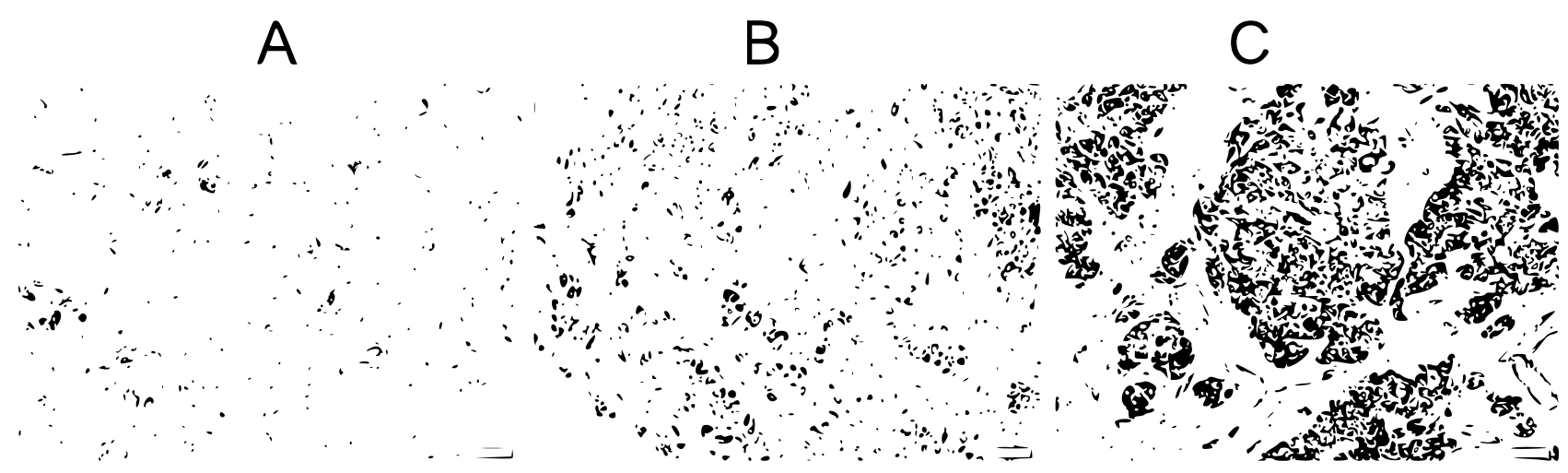

Figure I Representative immunohistochemical staining sections for ROMOI with different histologic scores $(\mathrm{H}$-scores) in adenocarcinoma $(\times 200)$. ROMOI was primarily detected in the cytoplasm of lung cancer cells. (A) H-score of 50; (B) H-score of I50; (C) H-score of 250. 
Table 2 Analyses Results For Progression-Free Survival And Overall Survival According To Clinical Parameters (N=49)

\begin{tabular}{|c|c|c|c|c|c|c|c|c|}
\hline & \multirow{2}{*}{$\begin{array}{l}\text { Median PFS } \\
\text { (Months) }\end{array}$} & \multirow{2}{*}{$\begin{array}{l}\text { Univariate } \\
p \text {-value }\end{array}$} & \multicolumn{2}{|c|}{ Multivariate } & \multirow{2}{*}{$\begin{array}{l}\text { Median OS } \\
\text { (Months) }\end{array}$} & \multirow{2}{*}{$\begin{array}{l}\text { Univariate } \\
p \text {-value }\end{array}$} & \multicolumn{2}{|c|}{ Multivariate } \\
\hline & & & $p$-value & $\mathrm{HR}, 95 \% \mathrm{Cl}$ & & & $p$-value & HR, 95\% Cl \\
\hline All & 10.4 & & & & 24.3 & & & \\
\hline $\begin{array}{l}\text { Age (years) } \\
\quad<70 \\
\geq 70\end{array}$ & $\begin{array}{l}16.1 \\
9.4\end{array}$ & 0.093 & 0.094 & $\begin{array}{l}\text { Reference } \\
2.06(0.88-4.8 I)\end{array}$ & $\begin{array}{l}24.0 \\
28.0\end{array}$ & 0.811 & NA & \\
\hline $\begin{array}{l}\text { Sex } \\
\text { Female } \\
\text { Male }\end{array}$ & $\begin{array}{l}10.4 \\
7.8\end{array}$ & 0.935 & NA & & $\begin{array}{l}24.0 \\
28.0\end{array}$ & 0.882 & NA & \\
\hline $\begin{array}{l}\text { Histology } \\
\text { SQCC } \\
\text { ADC }\end{array}$ & $\begin{array}{l}12.2 \\
9.4\end{array}$ & 0.613 & NA & & $\begin{array}{l}23.8 \\
22.0\end{array}$ & 0.534 & NA & \\
\hline $\begin{array}{c}\text { Stage } \\
\text { IIIA } \\
\text { IIIB }\end{array}$ & $\begin{array}{l}12.2 \\
9.4\end{array}$ & 0.174 & 0.730 & $\begin{array}{l}\text { Reference } \\
1.16(0.3-2.54)\end{array}$ & $\begin{array}{l}24.0 \\
23.7\end{array}$ & 0.505 & NA & \\
\hline $\begin{array}{l}\text { ECOG } \\
\text { performance } \\
0-1 \\
2\end{array}$ & $\begin{array}{l}10.8 \\
10.0\end{array}$ & 0.695 & NA & & $\begin{array}{l}32.5 \\
17.0\end{array}$ & 0.046 & 0.025 & $\begin{array}{l}\text { Reference } \\
2.37 \text { (1.08-6.55) }\end{array}$ \\
\hline $\begin{array}{l}\text { Smoking status } \\
\text { Current } \\
\text { Former or never }\end{array}$ & $\begin{array}{l}9.4 \\
12.1\end{array}$ & 0.053 & 0.048 & $\begin{array}{l}2.03(1.01-4.46) \\
\text { Reference }\end{array}$ & $\begin{array}{l}21.0 \\
42.5\end{array}$ & 0.102 & $0.06 \mathrm{I}$ & $\begin{array}{l}\text { I.45 (0.53-4.32) } \\
\text { Reference }\end{array}$ \\
\hline $\begin{array}{l}\text { Radiotherapy } \\
\text { technique } \\
\text { IMRT } \\
\text { 3D-CRT }\end{array}$ & $\begin{array}{l}12.2 \\
10.0\end{array}$ & 0.358 & NA & & $\begin{array}{l}21.0 \\
18.4\end{array}$ & 0.384 & NA & \\
\hline $\begin{array}{l}\text { Total dose (BED, } \\
\text { Gy,0) } \\
\quad<76 \\
\geq 76\end{array}$ & $\begin{array}{l}9.2 \\
12.2\end{array}$ & 0.246 & 0.487 & $\begin{array}{l}\text { I.39 (0.55-3.56) } \\
\text { Reference }\end{array}$ & $\begin{array}{l}24.0 \\
24.2\end{array}$ & 0.752 & NA & \\
\hline $\begin{array}{l}\text { Daily dose (Gy) } \\
\quad \leq 2 \\
>2\end{array}$ & $\begin{array}{l}10.0 \\
12.3\end{array}$ & 0.151 & 0.831 & $\begin{array}{l}1.12(0.49-3.11) \\
\text { Reference }\end{array}$ & $\begin{array}{l}24.9 \\
33.0\end{array}$ & 0.296 & 0.554 & $\begin{array}{l}\text { I.27 (0.57-2.82) } \\
\text { Reference }\end{array}$ \\
\hline $\begin{array}{l}\text { Radiotherapy } \\
\text { interruption } \\
\text { Yes } \\
\text { No }\end{array}$ & $\begin{array}{l}10.2 \\
12.4\end{array}$ & 0.975 & NA & & $\begin{array}{l}24.0 \\
28.1\end{array}$ & 0.432 & NA & \\
\hline $\begin{array}{l}\text { Chemotherapy } \\
\text { Yes } \\
\text { No }\end{array}$ & $\begin{array}{l}10.4 \\
10.2\end{array}$ & 0.683 & NA & & $\begin{array}{l}31.6 \\
23.9\end{array}$ & 0.712 & NA & \\
\hline $\begin{array}{l}\text { Romol } \\
\text { expression } \\
\text { Low } \\
\text { High }\end{array}$ & $\begin{array}{l}12.2 \\
7.0\end{array}$ & 0.054 & 0.041 & $\begin{array}{l}\text { Reference } \\
\text { I.87 (I.02-4.23) }\end{array}$ & $\begin{array}{l}29.0 \\
13.5\end{array}$ & 0.038 & 0.026 & $\begin{array}{l}\text { Reference } \\
2.79(1.13-6.87)\end{array}$ \\
\hline
\end{tabular}

Abbreviations: ECOG, Eastern Cooperative Oncology Group; ADC, adenocarcinoma; SQCC, squamous cell carcinoma; IMRT, intensity-modulated radiotherapy; 3D-CRT, 3-dimensional conformal radiotherapy; $\mathrm{BED}$, biologically equivalent dose; PFS, progression-free survival; OS, overall survival; $\mathrm{HR}$, hazard ratio; $\mathrm{Cl}$, confidence interval. 

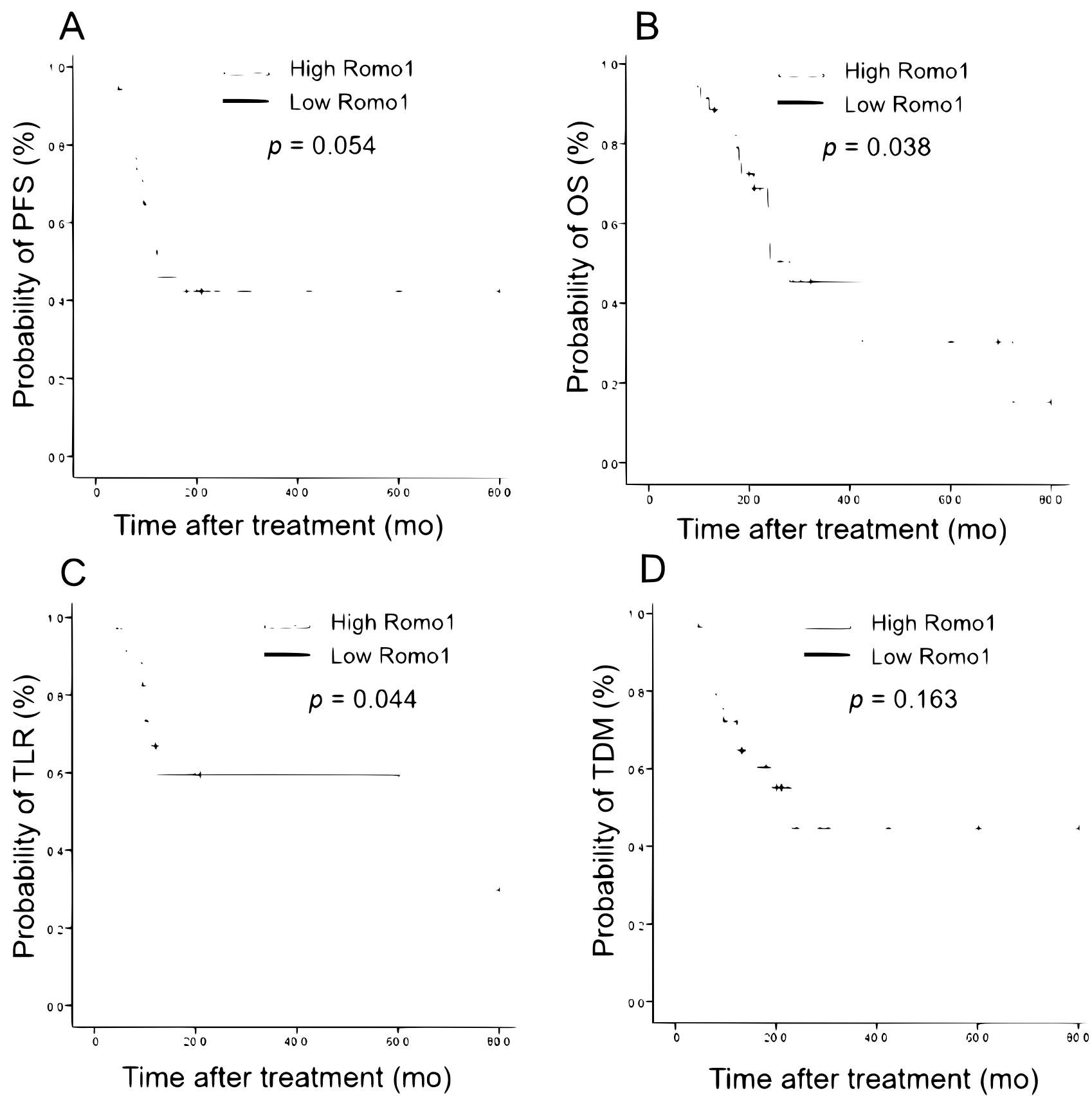

Figure 2 Kaplan-Meier survival curves for (A) progression-free survival (PFS), (B) Overall survival (OS), (C) Time to loco-regional recurrence (TLR), and (D) Time to distant metastasis (TDM) in all patients. $P$ values were determined using the log rank test.

experienced concurrent distant metastases. Of those 12 patients, $8(8 / 12,66.7 \%)$ received palliative chemotherapy. Among the 10 patients $(10 / 22,45.5 \%)$ who experienced loco-regional recurrence without distant metastasis, 1 received salvage surgical resection, 1 received salvage surgical resection followed by chemotherapy, 4 received salvage RT, 2 received salvage RT followed by chemotherapy, and 2 refused salvage treatment.
Results for TLR and TDM according to clinicopathological parameter are summarized in Table 3. The median TLR for the overall population was 38.5 months (range, 3.6-80.0 months). In univariate analysis, advanced age and RT without chemotherapy showed a non-significant decreasing trend for TLR, and high ROMO1 expression was significantly associated with shorter TLR (10.2 versus 60.4 months, $p=0.044)$. In the multivariate analysis, RT 
Table 3 Analyses Results For Time To Loco-Regional Recurrence And Time To Distant Metastases According To Clinical Parameters $(\mathrm{N}=49)$

\begin{tabular}{|c|c|c|c|c|c|c|c|c|}
\hline & \multirow{2}{*}{$\begin{array}{l}\text { Median } \\
\text { TLR } \\
\text { (Months) }\end{array}$} & \multirow{2}{*}{$\begin{array}{l}\text { Univariate } \\
p \text {-value }\end{array}$} & \multicolumn{2}{|c|}{ Multivariate } & \multirow{2}{*}{$\begin{array}{l}\text { Median } \\
\text { TDM } \\
\text { (Months) }\end{array}$} & \multirow{2}{*}{$\frac{\text { Univariate }}{p \text {-value }}$} & \multicolumn{2}{|c|}{ Multivariate } \\
\hline & & & $p$-value & $\mathrm{HR}, 95 \% \mathrm{Cl}$ & & & $p$-value & $\mathrm{HR}, 95 \% \mathrm{Cl}$ \\
\hline All & 38.5 & & & & 18.5 & & & \\
\hline $\begin{array}{l}\text { Age (years) } \\
\quad<70 \\
\geq 70\end{array}$ & $\begin{array}{l}55.4 \\
18.6\end{array}$ & 0.079 & 0.063 & $\begin{array}{l}\text { Reference } \\
2.36(0.96-5.82)\end{array}$ & $\begin{array}{l}23.0 \\
16.2\end{array}$ & 0.516 & NA & \\
\hline $\begin{array}{l}\text { Sex } \\
\qquad \text { Male } \\
\text { Female }\end{array}$ & $\begin{array}{l}60.4 \\
11.0\end{array}$ & 0.419 & NA & & $\begin{array}{l}20.5 \\
15.1\end{array}$ & 0.863 & NA & \\
\hline $\begin{array}{l}\text { Histology } \\
\text { SQCC } \\
\text { ADC }\end{array}$ & $\begin{array}{l}40.4 \\
21.2\end{array}$ & 0.507 & NA & & $\begin{array}{l}22.0 \\
13.2\end{array}$ & 0.687 & NA & \\
\hline $\begin{array}{c}\text { Stage } \\
\text { IIIA } \\
\text { IIIB }\end{array}$ & $\begin{array}{l}58.5 \\
10.8\end{array}$ & 0.293 & 0.622 & $\begin{array}{l}\text { Reference } \\
1.26(0.5 \mid-3.11)\end{array}$ & $\begin{array}{l}24.1 \\
12.2\end{array}$ & $0.44 I$ & NA & \\
\hline $\begin{array}{l}\text { ECOG } \\
\text { performance } \\
0-1 \\
2\end{array}$ & $\begin{array}{l}58.5 \\
12.0\end{array}$ & 0.925 & NA & & $\begin{array}{l}20.6 \\
14.7\end{array}$ & 0.394 & NA & \\
\hline $\begin{array}{l}\text { Smoking } \\
\text { status } \\
\text { Current } \\
\text { Former or } \\
\text { never }\end{array}$ & $\begin{array}{l}12.4 \\
55.1\end{array}$ & $0.28 \mathrm{I}$ & 0.238 & $\begin{array}{l}\text { I.75 (0.69-4.47) } \\
\text { Reference }\end{array}$ & $\begin{array}{l}16.5 \\
22.0\end{array}$ & 0.167 & 0.247 & $\begin{array}{l}\text { I.74 (0.68-4.44) } \\
\text { Reference }\end{array}$ \\
\hline $\begin{array}{l}\text { Radiotherapy } \\
\text { technique } \\
\text { IMRT } \\
\text { 3D-CRT }\end{array}$ & $\begin{array}{l}18.0 \\
48.2\end{array}$ & 0.757 & NA & & $\begin{array}{l}19.0 \\
20.1\end{array}$ & 0.339 & NA & \\
\hline $\begin{array}{l}\text { Total dose } \\
\text { (BED, Gy } 10) \\
\quad<76 \\
\geq 76\end{array}$ & $\begin{array}{l}16.0 \\
55.8\end{array}$ & 0.568 & NA & & $\begin{array}{l}15.1 \\
23.2\end{array}$ & 0.567 & NA & \\
\hline $\begin{array}{l}\text { Daily dose } \\
\text { (Gy) } \\
\leq 2 \\
>2\end{array}$ & $\begin{array}{l}15.3 \\
50.2\end{array}$ & 0.883 & NA & & $\begin{array}{l}18.0 \\
22.6\end{array}$ & 0.406 & NA & \\
\hline $\begin{array}{l}\text { Radiotherapy } \\
\text { interruption } \\
\text { Yes } \\
\text { No }\end{array}$ & $\begin{array}{l}10.8 \\
58.1\end{array}$ & 0.768 & NA & & $\begin{array}{l}16.1 \\
20.9\end{array}$ & 0.755 & NA & \\
\hline $\begin{array}{l}\text { Chemotherapy } \\
\text { Yes } \\
\text { No }\end{array}$ & $\begin{array}{l}55.2 \\
10.8\end{array}$ & 0.066 & 0.049 & $\begin{array}{l}\text { Reference } \\
2.86(1.01-5.43)\end{array}$ & $\begin{array}{l}22.4 \\
14.1\end{array}$ & 0.266 & 0.274 & $\begin{array}{l}\text { Reference } \\
\text { I.66 (0.67-4.08) }\end{array}$ \\
\hline
\end{tabular}

(Continued) 
Table 3 (Continued).

\begin{tabular}{|c|c|c|c|c|c|c|c|c|}
\hline & \multirow{2}{*}{$\begin{array}{l}\text { Median } \\
\text { TLR } \\
\text { (Months) }\end{array}$} & \multirow{2}{*}{$\begin{array}{l}\text { Univariate } \\
p \text {-value }\end{array}$} & \multicolumn{2}{|c|}{ Multivariate } & \multirow{2}{*}{$\begin{array}{l}\text { Median } \\
\text { TDM } \\
\text { (Months) }\end{array}$} & \multirow{2}{*}{$\begin{array}{l}\text { Univariate } \\
p \text {-value }\end{array}$} & \multicolumn{2}{|c|}{ Multivariate } \\
\hline & & & $p$-value & $\mathrm{HR}, 95 \% \mathrm{Cl}$ & & & $p$-value & $\mathrm{HR}, 95 \% \mathrm{Cl}$ \\
\hline $\begin{array}{l}\text { Romol } \\
\text { expression }\end{array}$ & & 0.044 & 0.042 & & & 0.163 & 0.253 & \\
\hline Low & 60.4 & & & Reference & 22.0 & & & Reference \\
\hline High & 10.2 & & & $2.71(1.04-6.28)$ & 15.5 & & & $1.73(0.43-5.10)$ \\
\hline
\end{tabular}

Abbreviations: ECOG, Eastern Cooperative Oncology Group; ADC, adenocarcinoma; SQCC, squamous cell carcinoma; IMRT, intensity-modulated radiotherapy; 3D-CRT, 3-dimensional conformal radiotherapy; BED, biologically equivalent dose; TLR, time to loco-regional recurrence; TDM, time to distant metastasis; HR, hazard ratio; CI, confidence interval.

without chemotherapy was significantly associated with shorter TLR (HR=2.86; 95\% CI: 1.01-5.43). In addition, high ROMO1 expression was independently associated with shorter TLR (HR=2.71, 95\% CI: 1.04-6.28). The Kaplan-Meier survival curves also showed that patients with high ROMO1 expression were likely to have shorter TLR (Figure 2C).

The median TDM for the overall population was 18.5 months (range, 2.5-80.0 months). The univariate and multivariate analyses indicated that there were no clinicopathological parameters associated with TDM. In addition, ROMO1 expression was not associated with TDM. The Kaplan-Meier survival curves for TDM are presented in Figure 2D.

\section{Discussion}

The present data demonstrate that ROMO1 overexpression is significantly associated with poor PFS and OS in patients with stage III NSCLC who received definitive RT. The association was also shown with TLR but not with TDM, suggesting that ROMO1 is more relevant to local recurrence rather than distance metastases. To the best of our knowledge, this is the first study demonstrating a relationship between ROMO1 expression and survival outcome in cancer patients who were treated with RT, which suggests that ROMO1 could have clinical usefulness as a potential prognostic biomarker in such patients.

Identifying resistance mechanisms or biomarkers for treatment response or survival is crucial to select high-risk patients and to increase the survival of those patients. For metastatic NSCLC, although clinically applicable biomarkers are lacking, numerous studies have been conducted on the potential biomarkers, such as genetic abnormality, including the KRAS mutation; ${ }^{14}$ DNA repair mechanisms, including excision repair of cross-complementation group $1 ;^{15}$ oncoproteins, including carcinoembryonic antigen (CEA) and cytokeratin 19 fragments (CYFRA 21-1); ${ }^{16}$ and inflammatory markers, including certain cytokines or relative ratios of blood cells. ${ }^{17,18}$ In contrast, for inoperable stage III disease that should be treated with RT, there are few biomarker studies. One study has reported that baseline leukocytosis and neutrophilia were associated with poor OS, PFS, TLR, and TDM in stage III NSCLC treated with concurrent CRT. ${ }^{19}$ Another study has demonstrated that blood-based biomarkers including osteopontin and CYFRA 21-1, alpha-2-macroglobulin, interleukin-2 receptor, and vascular endothelial growth factor showed predictive value in patients with inoperable stage I-IIIB NSCLC. ${ }^{20}$ These studies require validation, and there is no prognostic biomarker with confirmed clinical usefulness for lung cancer patients treated with RT.

Studies have demonstrated that ROS are critically involved in many steps of cancer development. The imbalance between pro-oxidants and anti-oxidants can result in oxidative stress, which is linked with DNA damage, carcinogenesis, and cancer progression. ${ }^{21,22}$ In addition, increased ROS level has been observed in many tumor cells. ${ }^{23}$ However, the exact mechanisms of intracellular ROS production and regulation were not clearly understood until recently. ROMO1 is a mitochondrial inner membrane protein known to regulate mitochondrial ROS production and is an essential redox sensor in mitochondrial dynamics. ${ }^{9}$ A very recent study showed that ROMO1 forms a viroporin-like nonselective cation channel that is inhibited by $\mathrm{Fe}^{2+}$ ions, a transition ion involved in ROS metabolism. ${ }^{24}$ Clinical studies have consistently suggested that this channel protein is associated with early recurrence and worse survival of surgically-treated human malignancies including lung cancer. ${ }^{10,11,13,25}$ Moreover, it has been reported that ROMO1 overexpression is associated with treatment response and survival in NSCLC patients who were treated with platinum-based chemotherapy. ${ }^{12}$ Thus, ROMO1 has emerged as a novel biomarker in cancer 
management. As RT-induced cell death results mainly from ROS-induced DNA damage, we postulated that ROMO1, a key regulator of intracellular ROS, could be involved in resistance to RT. The aforementioned studies and our data have demonstrated that ROMO1 overexpression is significantly associated with poor survival outcome in cancer patients regardless of treatment modality. Although further large-scale prospective studies are required, the consistent results suggest that ROMO1 could be a potential prognostic biomarker for cancer management in various clinical settings.

The exact mechanism of how ROMO1 is related to treatment resistance and shorter survival in stage III NSCLC treated with RT is unclear. However, enhanced adaptation to oxidative stress by ROMO1 could be a possible answer. ROMO1-induced ROS production is indispensable for proliferation of cancer and normal cells, which might result from upregulation of various self-protective anti-oxidants. ${ }^{9}$ Thus, high ROMO1 expression can be conferred to a well-adapted state or resistance of DNA damage against external stress including irradiation or chemotherapy. Moreover, ROMO1-related persistent oxidative stress can increase cancer cell aggressiveness and invasiveness by inducing continuous genomic instability. ${ }^{21,26,27}$ Although further preclinical studies are required to confirm our hypothesis, it is supported by a previous study reporting that ROMO1 is associated with sensitivity to $\gamma$-radiation in lung cancer cells. $^{28}$

This study has several limitations. First, as this study is retrospective in nature, there may be inherent bias. For example, RT fractionation schedules and RT techniques were decided by the attending radiation oncologist rather than using a pre-determined definite protocol, which may affect clinical outcome. Second, this is a small explorative study performed in a single institution. To make up for the small sample size, we analyzed various survival outcome parameters including TLR and TDM simultaneously. Third, we only evaluated tissue ROMO1 expression using immunohistochemical staining. Future validation studies should evaluate data on serum or plasma ROMO1 level as well as those from tissues using various methods including Western blot, reverse transcription polymerase chain reaction, or enzyme-linked immunosorbent assay. Fourth, the treatment modalities used in the study were somewhat heterogeneous; for instance, patients who received RT alone or CRT were analyzed together. However, this may reflect current realworld practice for stage III NSCLC, and we successfully demonstrated the association between ROMO1 and RT outcome despite the heterogeneity. Finally, we did not evaluate or compare the clinical utility of other biomarkers. As previous studies have suggested, ${ }^{29,30}$ a comparison with diagnostic value or an investigation of the combined effect with established biomarkers or serum ROMO1 would provide more informative data.

\section{Conclusion}

In summary, we verified that ROMO1 may be associated with an unfavorable clinical outcome in stage III NSCLC patients who were treated with RT. Our results are in accordance with previous studies conducted in different clinical settings and confirm the potential usefulness of ROMO1 as a prognostic biomarker. If large-scale prospective studies confirm our results, ROMO1 might help us to accomplish precision medicine in an RT setting by selecting high-risk patients who may require additional consolidation treatment and a closer follow-up. In addition, the consistent data between ROMO1 and clinical outcome provide a basis for future investigation using other clinical specimens such as serum or bronchoalveolar lavage fluid and for other treatment modalities including targeted therapy or immunotherapy, which might facilitate clinical use of this novel protein.

\section{Acknowledgments}

This research was supported by a grant from the Korea Health Technology R\&D Project through the Korea Health Industry Development Institute, funded by the Ministry of Health \& Welfare (HI18C1944) and by a grant from the Basic Research Program through the National Research Foundation funded by the Ministry of Science and ICT (2019R1F1A1041812) of Republic of Korea, and was supported by the Kyung Hee University Research Fund in 2018 (KHU-20181675).

\section{Disclosure}

The authors declare that they have no competing interests in this work.

\section{References}

1. Jung KW, Won YJ, Kong HJ, Lee ES. Cancer statistics in Korea: incidence, mortality, survival, and prevalence in 2015. Cancer Res Treat. 2018;50(2):303-316. doi:10.4143/crt.2018.143

2. Jemal A. Global burden of cancer: opportunities for prevention. Lancet. 2012;380(9856):1797-1799. doi:10.1016/S0140-6736(12)616 $88-2$ 
3. Kim HC, Jung CY, Cho DG, et al. Clinical characteristics and prognostic factors of lung cancer in Korea: a pilot study of data from the korean nationwide lung cancer registry. Tuberc Respir Dis (Seoul). 2019;82(2):118-125. doi:10.4046/trd.2017.0128

4. Siegel RL, Miller KD, Jemal A. Cancer statistics, 2015. sdfj. 2015;65 (1):5-29. doi:10.3322/caac. 21254

5. Yamamoto N, Nakagawa K, Nishimura Y, et al. Phase III study comparing second- and third-generation regimens with concurrent thoracic radiotherapy in patients with unresectable stage III nonsmall-cell lung cancer: West Japan Thoracic Oncology Group WJTOG0105. J Clin Oncol. 2010;28(23):3739-3745. doi:10.1200/ JCO.2009.24.5050

6. Ahn JS, Ahn YC, Kim JH, et al. Multinational randomized phase III trial with or without consolidation chemotherapy using docetaxel and cisplatin after concurrent chemoradiation in inoperable stage III nonsmall-cell lung cancer: KCSG-LU05-04. J Clin Oncol. 2015;33 (24):2660-2666. doi:10.1200/JCO.2014.60.0130

7. Senan S, Brade A, Wang LH, et al. PROCLAIM: randomized phase III trial of pemetrexed-cisplatin or etoposide-cisplatin plus thoracic radiation therapy followed by consolidation chemotherapy in locally advanced nonsquamous non-small-cell lung cancer. J Clin Oncol. 2016;34(9):953-962. doi:10.1200/JCO.2015.64.8824

8. Hanna N, Neubauer M, Yiannoutsos C, et al. Phase III study of cisplatin, etoposide, and concurrent chest radiation with or without consolidation docetaxel in patients with inoperable stage III nonsmall-cell lung cancer: the hoosier oncology group and U.S. Oncology. J Clin Oncol. 2008;26(35):5755-5760. doi:10.1200/JCO. 2008.17.7840

9. Chung YM, Kim JS, Yoo YD. A novel protein, ROMO1, induces ROS production in the mitochondria. Biochem Biophys Res Commun. 2006;347(3):649-655. doi:10.1016/j.bbrc.2006.06.140

10. Chung JS, Park S, Park SH, et al. Overexpression of ROMO1 promotes production of reactive oxygen species and invasiveness of hepatic tumor cells. Gastroenterology. 2012;143(4):1084-1094 e1087. doi:10.1053/j.gastro.2012.06.038

11. Kim HJ, Jo MJ, Kim BR, et al. Reactive oxygen species modulator-1 (ROMO1) predicts unfavorable prognosis in colorectal cancer patients. PLoS One. 2017;12(5):e0176834. doi:10.1371/journal.pone. 0176834

12. Lee SH, Choi SI, Lee JS, et al. Reactive Oxygen Species Modulator 1 (ROMO1) predicts poor outcomes in advanced non-small cell lung cancer patients treated with platinum-based chemotherapy. Cancer Res Treat. 2017;49(1):141-149. doi:10.4143/crt.2016.133

13. Lee SH, Min JW, Lee JS, et al. Reactive oxygen species modulator 1 (ROMO1) overexpression is an independent predictor of poor survival in NSCLC patients who undergo surgical resection. Lung Cancer. 2015;87(1):45-52. doi:10.1016/j.lungcan.2014.11.004

14. Roberts PJ, Stinchcombe TE. KRAS mutation: should we test for it, and does it matter? J Clin Oncol. 2013;31(8):1112-1121. doi:10. 1200/JCO.2012.43.0454

15. Cobo M, Isla D, Massuti B, et al. Customizing cisplatin based on quantitative excision repair cross-complementing 1 mRNA expression: a phase III trial in non-small-cell lung cancer. J Clin Oncol. 2007;25(19):2747-2754. doi:10.1200/JCO.2006.09.7915
16. Lee JH, Chang JH. Diagnostic utility of serum and pleural fluid carcinoembryonic antigen, neuron-specific enolase, and cytokeratin 19 fragments in patients with effusions from primary lung cancer. Chest. 2005;128(4):2298-2303. doi:10.1378/chest.128.4.2298

17. Zhao QT, Yuan Z, Zhang H, et al. Prognostic role of platelet to lymphocyte ratio in non-small cell lung cancers: a meta-analysis including 3,720 patients. Int $J$ Cancer. 2016;139(1):164-170. doi:10.1002/ijc. 30060

18. Templeton AJ, McNamara MG, Seruga B, et al. Prognostic role of neutrophil-to-lymphocyte ratio in solid tumors: a systematic review and meta-analysis. J Natl Cancer Inst. 2014;106(6):dju124. doi:10. 1093/jnci/dju061

19. Schernberg A, Mezquita L, Boros A, et al. Neutrophilia as prognostic biomarker in locally advanced stage III lung cancer. PLoS One. 2018;13(10):e0204490. doi:10.1371/journal.pone.0204490

20. Carvalho S, Troost EG, Bons J, Menheere P, Lambin P, Oberije C. Prognostic value of blood-biomarkers related to hypoxia, inflammation, immune response and tumour load in non-small cell lung cancer a survival model with external validation. Radiother Oncol. 2016;119 (3):487-494. doi:10.1016/j.radonc.2016.04.024

21. Sosa V, Moline T, Somoza R, Paciucci R, Kondoh H, LL ME. Oxidative stress and cancer: an overview. Ageing Res Rev. 2013;12 (1):376-390. doi:10.1016/j.arr.2012.10.004

22. Finkel T, Holbrook NJ. Oxidants, oxidative stress and the biology of ageing. Nature. 2000;408(6809):239-247. doi:10.1038/35041687

23. Prasad S, Gupta SC, Tyagi AK. Reactive oxygen species (ROS) and cancer: role of antioxidative nutraceuticals. Cancer Lett. 2017;387:95105. doi:10.1016/j.canlet.2016.03.042

24. Lee GY, You DG, Lee HR, Hwang SW, Lee CJ, Yoo YD. ROMO1 is a mitochondrial nonselective cation channel with viroporin-like characteristics. J Cell Biol. 2018;217(6):2059-2071. doi:10.1083/jcb.20 1709001

25. Kim HJ, Jo MJ, Kim BR, et al. Overexpression of ROMO1 is an unfavorable prognostic biomarker and a predictor of lymphatic metastasis in non-small cell lung cancer patients. Onco Targets Ther. 2018;11:4233-4246. doi:10.2147/OTT.S161587

26. Toyokuni S, Okamoto K, Yodoi J, Hiai H. Persistent oxidative stress in cancer. FEBS Lett. 1995;358(1):1-3. doi:10.1016/0014-5793(94) 01368-b

27. Wu WS. The signaling mechanism of ROS in tumor progression. Cancer Metastasis Rev. 2006;25(4):695-705. doi:10.1007/s10555006-9037-8

28. Kim IG, Kim SY, Kim HA, et al. Disturbance of DKK1 level is partly involved in survival of lung cancer cells via regulation of ROMO1 and gamma-radiation sensitivity. Biochem Biophys Res Commun 2014;443(1):49-55. doi:10.1016/j.bbrc.2013.11.038

29. Lee SH, Lee JS, Lee EJ, et al. Serum reactive oxygen species modulator 1 (ROMO1) as a potential diagnostic biomarker for non-small cell lung cancer. Lung Cancer. 2014;85(2):175-181. doi:10.1016/j.lungcan. 2014.05.023

30. Lee SH, Park MJ, Choi SI, Lee EJ, Lee SY, In KH. Reactive oxygen species modulator 1 (ROMO1) as a novel diagnostic marker for lung cancer-related malignant effusion. Medicine (Baltimore). 2017;96(4): e5975. doi:10.1097/MD.0000000000005975

OncoTargets and Therapy

Dovepress

\section{Publish your work in this journal}

OncoTargets and Therapy is an international, peer-reviewed, open access journal focusing on the pathological basis of all cancers, potential targets for therapy and treatment protocols employed to improve the management of cancer patients. The journal also focuses on the impact of management programs and new therapeutic

agents and protocols on patient perspectives such as quality of life, adherence and satisfaction. The manuscript management system is completely online and includes a very quick and fair peer-review system, which is all easy to use. Visit http://www.dovepress.com/ testimonials.php to read real quotes from published authors.

Submit your manuscript here: https://www.dovepress.com/oncotargets-and-therapy-journal 